\title{
A Constructive Intrusion Detection System for Preventing Attacks in Mobile ADHOC Networks
}

\author{
T. Kamaleshwar \\ Research Scholar (CSE) \\ Annamalai University \\ Tamilnadu, India
}

\author{
K. Venkatachalapathy, $\mathrm{PhD}$ \\ Professsor (CIS) \\ Annamalai University \\ Tamilnadu, India
}

\begin{abstract}
Mobile Adhoc Network (MANET) is an independent system of mobile nodes connected by wireless system. The nodes are free to change dynamically and it can change the topology for the requirement. It establishing an efficient and ideal route between the communicating nodes is the elemental concern of the routing protocols of MANET. Each node not only acts as end system, but also acts as a router to forward packets. In MANET it is very difficult to design the robust security solution for various attacks. Here we are analyzing overall performance as well as the security of the Intrusion Detection system. We propose a Constructive Intrusion detection system based on the network and host based system. First of all it provided maximum security, it supports high scalability and high availability, and it provides best result on both normal and abnormal behaviors of different packets. The proposed model includes integration of individual model to produced batter results.
\end{abstract}

\section{Keywords}

Intrusion Detection System, Network detection system, Host based detection system.

\section{INTRODUCTION}

Now-a-days the number of networks keeps growing in parallel with dealing, especially on the Internet while chatting, video conferencing, live streaming, surfing, etc. These various types of transactions bring in many anomalies and intrusion into the computer network. The network traffic is mostly seen to display sudden abnormal behaviour form the system. Some of these aberrations are engendered by malicious network attacks such as Denial-Of-Service or virus, whereas others are the result of equipment failures and accidental outages [2]. Many methods that have been developed by outsourcing and play vital roles to secure network infrastructure and communication via the Internet such as through the use of anti-virus, firewalls, software package and intrusion detection systems. The Intrusion detection system is where comes by software and hardware resource, where it will detect the malicious node in the network structure. The current firewalls cannot defend against every category of intrusion, whereby some intrusions take advantages of computer system vulnerabilities [4]. An Intrusion Detection system (IDS) it provides network observation and is an extra wall to secure the network around-the-clock. The Intrusion detection system is a process of examine an intrusion into a system through the analysing of available information concerning the state of the network system, it monitors the user activities and reporting to a management station network. Intrusion detection system refers to the detection of brutal activity (penetrations, breakins and other forms of network blackguard in a computerrelated system [7]. Therefore, the Intrusion detection techniques are classified into Network based and Host based depending upon the case and source of information used to identify security breaches. The definition from the study that an intrusion is any activity that changes a system from a safe state to an unsafe condition, but this does little to clear up the situation. Another definition declares, in core, that an intrusion is anything that breach the policy of the site under any consideration, but this also does small to address the brings out at hand. Here by determine each word of Intrusion detection (ID).

Intrusion: The move of wrongful entering upon, compassing or taking possession of the property of another.

Detection: The act of determining or discovering the presence, existence or fact of.

Intrusion Detection: The act of determining or discovering the presence, existence or fact of the wrongful entering upon, compassing or taking possession of the property of another. [10]

Table 1: Types of attack

\begin{tabular}{|c|c|c|}
\hline S.No & Type of attack & Parameters \\
\hline 1 & Black hole & $\begin{array}{l}\text { 1.Buffer size } \\
\text { 2.Packet delivery ratio }\end{array}$ \\
\hline 2. & Wormhole & $\begin{array}{l}\text { 1.Buffer size } \\
\text { 2.Packet delivery ratio } \\
\text { 3.Location estimation } \\
\text { 4.time to leave }\end{array}$ \\
\hline 3. & Gray hole & 1.Packet delivery ratio \\
\hline 4. & $\begin{array}{l}\text { Denial of Service } \\
\text { attack }\end{array}$ & $\begin{array}{l}\text { 1.Energy consumption } \\
\text { 2.Control message } \\
\text { 3.Packet delivery ratio } \\
\text { 4.Buffer size }\end{array}$ \\
\hline
\end{tabular}

In MANET normally the attacks are classified into two. One is Passive and Active attacks, where the passive attack it gather the information and will not disturb to the network. Whereas in active attacks, it modify/alter the data like inject the packets/drops the packet, by disturbing the network. When compare to passive attack, the active is very dangerous. Due to the mobility and open media nature the Mobile Adhoc networks are more prone to the security threats compared to the wired networks. Therefore security need to be tight in mobile networks compared to the wired. There is a need of comprehensive security solution which can deal with attack the various types of attack. There is also one more 
classification of attack in MANET, namely External attack and internal attack. The External attack carried out by nodes that were not belonging to the presence domain of the network. In Internal attacks are from compromised nodes, which are normally part of the network.

. The concept of security and the word Intrusion system might be restraining and complicated. The objective of this paper is to enhance a tool that mediates the user agent and the mechanism to reach the goals. The intrusion system was already existing techniques in platform. The people need to use the Intrusion system in order to find the attacks in network based system and host based system. The operations includes set of rules to find the attacks of outlanders to reach and read personal content that is located in our personal computer. The system is connected directly to the internet are tending to reliably attack and probing. The primary objective of the proposed work is to develop a new "Constructive Intrusion Detection System" (CIDS) which was including of both type network and host functionary.

\section{LITERATURE SURVEY}

Routing in MANET means to choose a best and proper path from source to destination. Routing means to choose a path. Routing terminology is used in different kinds of networks such as in electronic data networks, telephone technology, and in the internet network. Here work is more concern about routing in mobile ad hoc networks [6]. Routing protocols in mobile adhoc network means that the mobile nodes will look for a route or path to connect to each other and share the data packets to the nodes [9]. Protocols are the set of rules through which two or more devices (electronic gadgets, mobile nodes or computers) can communicate to each other. In mobile adhoc networks the routing is firmly done with the help of routing tables. These tables are kept in the memory cache of these mobile nodes [6]. When the routing process is going on, it will route the data packets in various mechanisms. The first one is unicast, in which it directly sends the data packets from the source to the destination. The second is multicast, in this the source node sends data packet to the exact multiple nodes in the network [6]. The third is broadcast; that means the source node sends a messages to all the nigh and far nodes in the network.

The routing has two general types, which are as under.

Static Routing: it was done by the administrator manually to forward the data packets in the network and it is permanent. No any other administrator can change this setting [5]. These static routers are well figured by the administrator, which means there is no need to make routing chart by the router itself

Dynamic Routing: is automatically done by the choice of router. It can route the traffic on any route depend on the routing table. Dynamic routing allows the routers to know about the networks and the interesting thing is to add this information in their routing tables. In dynamic routing the routers exchange the routing information if there is some change in the topology [6]. Exchanging information between these dynamic routers learn to know about the new routes and networks. Dynamic routing is more flexible than static routing. In dynamic routing it have the capability to overcome the overload traffic. Dynamic routing uses different paths to forward the data packets. Dynamic routing is better than static routing [6].

\section{SYSTEM OVERVIEW}

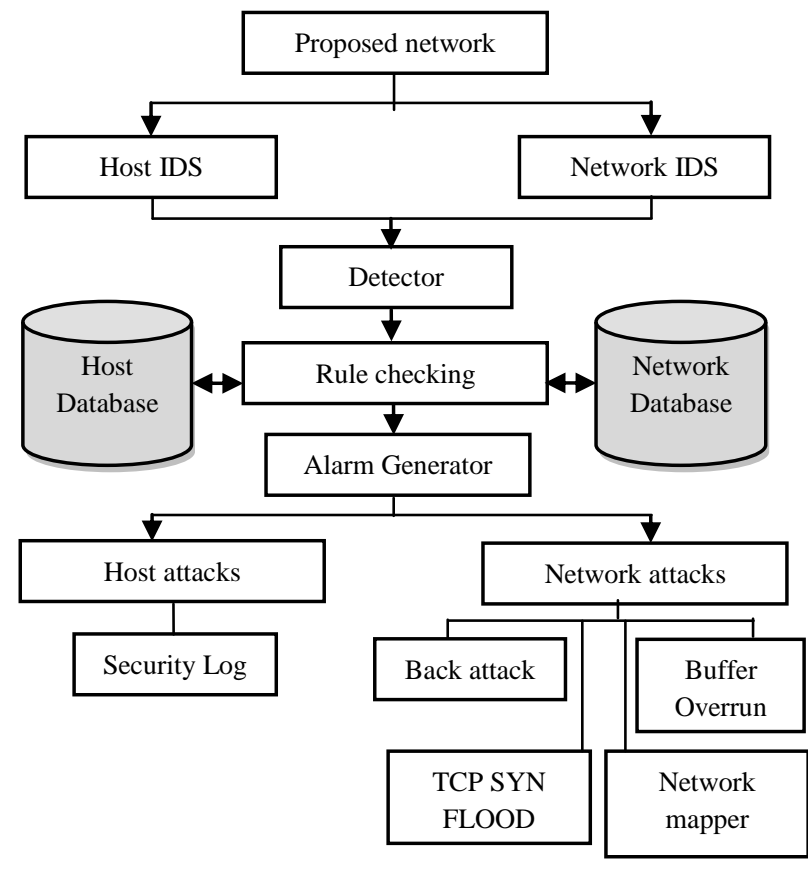

Figure 1: Combination of Host and Network IDS

In Figure 1 shows the architecture of the combination of Host based IDS and Network based IDS, from this it's known as Constructive Intrusion Detection system (CIDS). In this figure a specific IDS was capture the packets and accessing to the sensor agent where the detector agent pass the captivate packets to rule matching process where the rule for perfect check attacks criteria from the overall database, where we have already dispute and stored maximum rule to find attack. The rule checking process will be done from the local storage of both the Host and network database. From the rule checking process, the next alarm generating process. After completing this stage alarm will activate if any type of attack find in the appropriate packet otherwise it will be deactivate and this processes will continue till on the proposed system. If at all there is no attack was held, the process flow will goes on.

In figure 2, it represents Network Intrusion detection system. In this proposed block, where it will check the different conditions from various attack. The first stage where it monitors the attacks and captures the packets, where is passing over internet as public network.. Since it is a affected packets and it be should be mark as an attack node. Where another condition for Back attack type, where this type of attack it's relate to Denial of service attack category. Normally back attack find on physical layer and land attack find on application layers.

In this proposed Constructive Intrusion detection system were both type of attack detection is used, namely Network based intrusion system and Host based intrusion system . From TCP header is extracted and examined its types of attributes. If Reset (RST) flag find in its exact attribute, then that was not a normal packet and it will flow to the alarm generator to note at every time of TCP packets header extracting the system checks the arriving of new IP header. From the IP header it only selects only TCP protocol from the network. In Figure 2 is showing block diagram of proposed Network based intrusion detection system (NIDS) there are two modes of the 
proposed IDS one is Network based and another is Host based. In Network based intrusion system we are detecting four abnormality or four types of attack in the appropriate packets which is follows "Back land attack, TCP SYN FLOOD, Buffer overrun, abnormal packets and Network map per. In other type of host based where we can find one type of attack by examine the security event ledger file, that which was stored in local system. The two security event which was normally occur, "unauthorized accessing" and "Login failed". In figure 2 it represents the block system of Network Intrusion detection system.

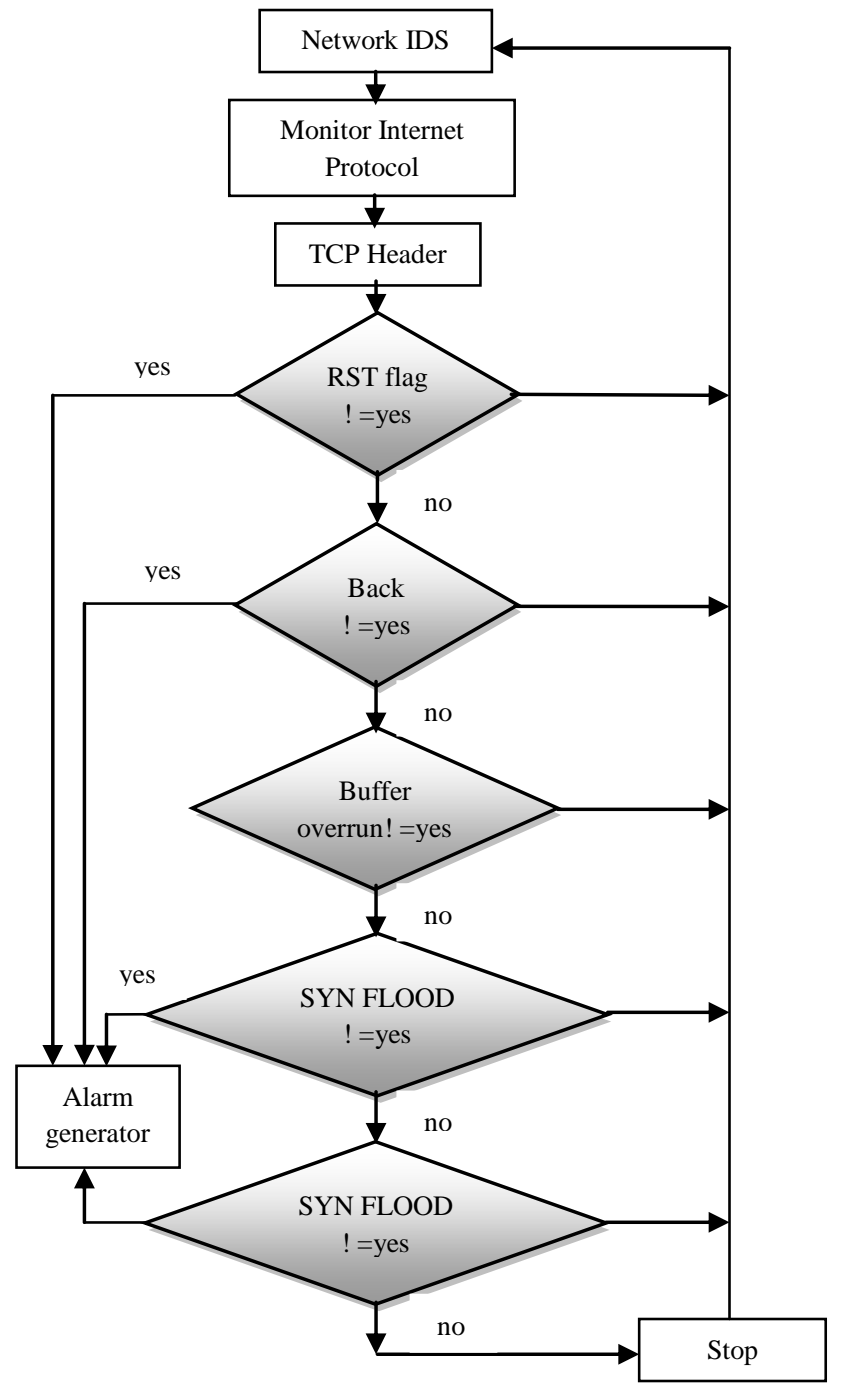

Figure 2: Work flow of NIDS

The consideration of these types of attack is to examine the number of Tcp packet which is arriving from the local host. In this system, we set a catch time. The time limit of 15 packets is $5 \mathrm{sec}$. If the host are sending more than 15 packets in the limited time of 5 seconds, then the second host is the intruder which is accumulating a report fake address then it will also said as an attack type.

The next condition for attack is buffer overrun or buffer overflow which is tends to UTR attack is unauthorized access from a unknown machine or remote machine. For this kind of attack we have to set a buffer size and check the overrun packets, if the buffer size is beyond the size, then the attack will be occurred. But, In case of packet size is big, for that the buffer size should be predefined. And the last one is TCPSYN
FLOOD attack, for this we defined a threshold value for the receiving packets, if the threshold is less than the set value is normal. Otherwise the value of threshold is increase, and then the network was affected by attack. The RST flag is nothing but of Reset flag, once the data is passed from the header, it will reset the flag for the impudent condition. The network intrusion detection system was carried out under these categories of attacks back attack, buffer overflow, TCP syn flood.

\section{METHODOLOGY}

In this part, we are going to see about the Host Intrusion detection system, where this system can be hybrid with both. In this system we have concentrated only for log in security, where the analyzer will detect the attack when the logging system enabled. Normally in internet world, the first security level everyone knows is Login password for a single user. Even that can prone to the hacker easily. The Host intrusion detection system is the methods of security analyser for networks and computers. In HIDS, where malware detection, antivirus, spyware-detection software are installed on every personal network computer, that has prone to the outside environment such as Internet. But, in network based system like anti threat is installed only at certain point node. Example like servers that which interface between the outsourcing environment and the segment to be safeguarded. By finding that types of attack, for that Host intrusion detection as been introduced. Where, HIDS will detect both attacks unauthorized and login/logoff time. This paper is focus on the both as Network and also Host based intrusion detection system. By the name suggests, that resides in a separate host system monitoring and testing on systems configuration. This extra layer of protection may ensure the past your firewall does not leave you into malicious act. HIDS has several aspects, such as anomaly detection, signature identification and detection analysis of protocol to protect you against malware threats.

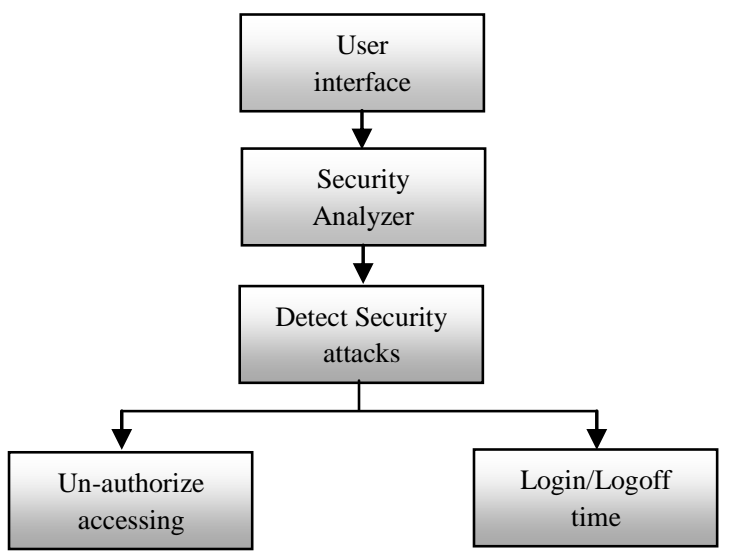

Figure 3: HIDS Architecture

In figure 3, where HIDS architecture is represented. This kind of intrusion detection system was prevented from the basic user host system. While us entering into the network, where the security analyzer will detect the security attacks from the Un-authorize users and Login/Login off time. As we know that in this log file there are so many values to examine the security of the system but we have concentrate only on two values which is already defined above. The proposed Host intrusion system call the security analyser to find or check attack in the local host then it will detect the attack in even log 
files. After completing this it will go to the alarm system for the information that this system was attacked from malicious nodes.

This paper focus on both of Host and the network intrusion system, to detect the attack we have set some parameter like buffer size for UTR, 3 IP address from same source in 1 second that means irrelevant address for TCP, DOS header flag are set Reset then abnormal and to detect the SYNLOOD attack. We are setting a threshold value for a time interval value of $\Delta \mathrm{T}$ which will be change from small to large. So we set $\Delta \mathrm{T}=10 \mathrm{sec}, 15 \mathrm{sec}, 20 \mathrm{sec}$ and $25 \mathrm{sec}$ and $\mathrm{T}=1000$ millisecond by default setting. The value of default will be use in the absence of actual threshold value, the HIDS have set password to the examined system and predefined a working time. For example we are entering into by using wrong user name and password and also login in wrong period of time to capture the host attack.

\section{PERFORMANCE EVALUATION}

For this experiment, we used our desktop machine. Configuration of that 2GB of RAM, windows XP SP2, $2.20 \mathrm{Ghz}$ where the performance is collected. The performance evaluation shows the comparison between the various attacks.

Table 2: Comparisons of attacks in N/W IDS

\begin{tabular}{|c|c|c|c|}
\hline $\begin{array}{ll}\text { No. } & \text { of } \\
\text { packets }\end{array}$ & Back attack & $\begin{array}{l}\text { Abnormal } \\
\text { activities }\end{array}$ & $\begin{array}{l}\text { Buffer } \\
\text { Overrun }\end{array}$ \\
\hline \multicolumn{4}{|c|}{ Presented Results are In Approx } \\
\hline 392 & 166 & 4 & 232 \\
\hline 736 & 652 & 9 & 682 \\
\hline 1144 & 1005 & 27 & 1060 \\
\hline
\end{tabular}

The above presented results for attack are in approximately. The number of packets are send from source to destination are listed, from the various attack analysis the results are observed. The network intrusion detection system that was defined from the four types of attacks, by setting the time limit to 5 seconds, TCP header was forwarded to the destination. By this we have listed the abnormal action in this network.

Table 3: TCP SYN Flood Attacks

\begin{tabular}{llll}
\hline S.No & $\begin{array}{l}\text { Time of capture } \\
\text { packets }\end{array}$ & TCP & $\begin{array}{l}\text { Number } \\
\text { attacks find by } \\
\text { CIDS }\end{array}$ \\
\hline Presented Results are In Approx & \\
\hline $\mathbf{1}$ & 10 seconds & \\
\hline $\mathbf{2}$ & 15 seconds & 08 \\
\hline $\mathbf{3}$ & 20 seconds & 27 \\
\hline
\end{tabular}

The TCP FLOOD attacks where various types of data's are forwarded on a period of time $(10 \mathrm{sec}, 15 \mathrm{sec}, 20 \mathrm{sec})$ from that we detect more number of malicious act in that network infrastructure. In this mention that routing as overhead and packet delivery ratio. In that, it founds the malicious node, so the hacker can continuously forwarding the packet easily and also overcomes the routing overhead.

\section{CONCLUSION}

In this paper, we proposed a constructive Intrusion detection system to detect various types of attacks like Land attack, TCP SYN FLOOD and Buffer overrun and non-typical packets in Host Intrusion detection and Network Intrusion detection. Finally for detecting the attacks in network a machine based intrusion detection system is implemented and simulated. The proposed Mobile Adhoc network CIDS system includes a new network infrastructure and attack detection technique additionally using. In Host IDS, we have determined the unauthorized accessing and login/logout failed. The proposed Constructive IDS as providing both type of functionality in a single system which is improving overall efficiency of the existing Intrusion detection system. In future we will cluster the Intrusion with cryptosystem like cryptography, Hash function and Digital signature. The cluster based Intrusion system that may work on layers protocol and try to find attacks on layers wise, in which layer what type of attack will execute and how we can prevent them.

\section{REFERENCES}

[1] Bo Sen, Kui Wu, vdo W. Pooch, "Zone-Based Intrusion Detection system for Mobile Ad Hoc Networks", journal on Adhoc Networks in gurd conference, Vol.10, No. 7, pp 1178-1190, March 2010.

[2] Chundong Wang, Quancai Deng, Qing Chang,Hua Zhang and Huaibin Wang "A New Intrusion Detection System Based on Protocol Acknowledgement" IEEE 2010.

[3] Meng L, Dipala WS, Grimm WM, inventors; Robert Bosch GmbaH, assignee. Dual sensing intrusion method and system with state-level fusion. United States patent US 7,262,696. 2007

[4] Jin-Tae Oh, Sang-Kil Park, Jong-Soo Jang and Yong- Hee Jeon "Detection of DDoS and IDS Evasion

Attacks in a High-Speed Networks Environment" published in IJCSNS International Journal of Computer Science and Network Security, VOL.7 No.6, June 2007

[5] Ajay Prakash Rai, Vineet, Rinkoo Bhatia, "Wormhole Attack Detection in Mobile Ad Hoc Networks", International Journal of Engineering and Innovative Technology, Vol.2, No. 3, pp 384-389, August 2012.

[6] Routing protocols and concepts, CCNA exploration companion guide. Introduction to AODV dynamic routing protocol. Chapter three, pp 148-177.

[7] Vera Marinova-Boncheva "A Short Survey of Intrusion Detection Systems" 2007

[8] Mod Alhamaety, Ali Yazdian "Intrusion Detection System Based On The Integrity of TCP Packet" published in World Academy of Science, Engineering and Technology 112007

[9] Loukas Lazos, and Marwan Krunz, "Selective Jamming/Dropping Insider Attacks in Wireless Mesh Networks" An International Journal on Engineering Science and Technology Arizona edu, Vol.2, No. 2, pp 265-269, April 2010.

[10] T. S. Sobhana "wireless intrusion detection system Classifications, good characteristics and state of- the- 
art”, Computer \& Interfaces 30, pp. 670-694, Science Direct, 2006.

[11] D. H. Denning, "An intrusion-detection model." IEEE Transactions on Software Engineering, Vol. SE-13(No. 2):222-232, Feb. 1987.

[12] Douglas J. Brown, Billy Suckow, and Tianqiu Wang "A Survey of Intrusion Detection Systems" 2004

[13] Rajiv Ranjan, Naresh Trivedi and Anop Srivastava, "Mitigating of Blackhole Attack in Manets", VSRD, International Journal of Computer Science and Information Tech., Vol.1, 53-57, 2011

[14] Maha Abdelhaq, Sami Serhan, Raed Alsaqour and Antoan Satrian, "Security Routing Mechanism for Black Hole Attack over AODV MANET Routing Protocol", Australian Journal of Basic and Applied Sciences,5:1137-1145, 2011.

[15] Ekta Kamboj,Harshil Rohil, "Detection of Black Hole Attack on AODV using Fuzzy Logic", Journal of Current Computer Science and Technology,Vol.1 Issue 6:316-318, 2011

[16] Mahesh Pal, "Multiclass Approaches for Support Vector Machine Based Land Cover Classification", 2008.

[17] Preeti Nagrath,Ashish Kumar,Shikha Bhardwaj, "Authenticated Routing Protocol based on Reputation System For Adhoc Networks", International Journal on Computer Science and Engineering, Vol.2: 3095-3099, 2010

[18] Adnan Nadeem, Michael P. Howarth," A Survey of MANET Intrusion Detection \& Prevention Approaches for Network Layer Attacks", IEEE communications surveys \& tutorials, Vol. 15, No. 4, Fifth quarter 2013
[19] Lung-Chung Li and Ru-Sheng Liua," Securing ClusterBased Ad Hoc Networks with Distributed Authorities", IEEE transactions on wireless system, VOL. 9, NO. 10, pp-3072-3081, OCTOBER 2011

[20] N. Nasser and Y. Chen, "Enhanced intrusion detection systems for discovering malicious nodes in mobile ad hoc network," in Proc. IEEE Int.Conf. Commun., Glasgow, Scotland, Jun. 24-28, 2007

[21] Asma Tuteja, Rajneesh Gujral, Sunil Thalia, -Comparative Performance Analysis of DSDV, AODV and DSR Routing Protocols in MANET using NS2\|, 2010 International Conference on Advances in Computer Engineering, 978-0-7695-4058-0/10 \$26.00 (C) 2010 IEEE.

[22] Martin K Parmar, Harikrishna B Jethva, - Survey on Mobile ADHOC Network and Security Attacks on Network Layer, International Journal of Advanced Research in Computer Science and Software Engineering, Volume 3, Issue 11, November 2013.

[23] Vikas Makram and Shrish Mohan Duby, A General Study of Associations rule mining in Intrusion Detection Systemll, International Journal of Emerging Technology and Advanced Engineering, ISSN 2250-2459, Volume 2, Issue 3, January 2012.

[24] Peng Zhan, Xinyu Yang, Wei Yu sa, and Xinwen Fu," A Loose Virtual Clustering Based Routing for Power Heterogeneous MANETs", IEEE Transactions on vehicular technology, VOL. 62, NO. 5, JUNE 2013 